\title{
Über Kapillardruck-Messungen normaler und veränderter Haut.
}

\author{
Von
}

\author{
Dr. Hugo Fasal, \\ Assistent der Abteilung.
}

Der Kapillardruck der menschlichen Haut wurde zuerst von v. Krie $\mathbf{s}^{1}$ ) bestimmt. N. von Kries hat im physiologischen Institut zu Leipzig den Blutdruck in den Kapillaren in der Weise bestimmt, daß er mit Hilfe belasteter Glasplättchen auf eine Fingerstelle einen Druck ausübte und das Gewicht ermittelte, welches notwendig war, um die komprimierte Haut blaß zu machen. v. Kries untersuchte den Kapillardruck bei verschiedener Haltung der Hände. Er fand den Blutdruck umso größer, je tiefer die Hände gehalten wurden. Eintauchen in heißes oder kaltes Wasser, Reizung durch den Indulstions-Strom, ließ keine Vermehrung des Blutdruckes erkennen; dagegen war eine nachweisbare Steigerung des Kapillardruckes durch Lompression der Venen (z. B. Umschnürung des Fingers) hervorzubringen.

Andere Untersuchungen als die genannten über den Kapillardruck in der menschlichen Haut liegen, soweit mir bekannt, bisher in der Literatur nicht vor, wiewol bei der Wichtigkeit dieses Gegenstandes Aufschlüsse hierüber sehr wünschenswert erschienen.

Ich bediente mich bei meinen Untersuchungen, welche den Zweck hatten, den Kapillardruck an verschiedenen nor-

1) Über den Druck in den Blutkapillaren der menschlichen Haut. Arbeiten aus der physiologischen Anstalt zu Leipzig. 1875. 10. Jahrgang. pag. 69. 
malen und krankhaft veränderten Hautstellen in Vergleichswerten zu bestimmen, des Kapillar-Manometers von Prof. S. v. Basch ${ }^{1}$ ). Mit Hilfe dieses Manometers wird der auf die Kapillaren wirkende Druck direkt, d. i. ohne Vermittlung eines Diaphragmas ausgeübt.

Bei allen derartigen Untersuchungen handelt es sich darum zu beobachten, bei welchem Druck, oder unter welcher Belastung die betreffende untersuchte Hautstelle blaß wird. Da es sich hiebei um Abschätzung feiner Farbenunterschiede handelt zeigen sich gewisse Fehlergrenzen.

Es wurden daher bei Messung des Kapillardrucks jeder einzelnen Hautstelle wiederholte Untersuchungen vorgenommen, von welchen dann der Durchschnittswert aufgezeichnet wurde.

Das Kapillar-Manometer von $\mathrm{B}$ a $\mathrm{s} \mathrm{ch}$ besteht aus einem Glastrichterchen, dessen obere Öffnung durch ein Gläschen verschlossen ist, und welches mit der schmäleren unteren Mündung anf die Haut aufgesetzt wird. Um den Abschluß luftdicht zu machen, wird auf den Rand. eine dünne Schichte Fischleim aufgetragen. Von diesem Trichter geht ein Glasröhrchen aus, das einerseits mit einem Manometer, andererseits mit einem Kautschukballon verbunden ist. Es wird nun das Trichterchen dicht auf die zu untersuchende Hautstelle aufgesetzt. Durch Druck auf den Kautschukballon wird eine Kompression der Luft erzeugt. Wäbrend ich durch das abschließende Gläschen hindurch das Blaßwerden der Haut beobachten kann, dient das Manometer dazu, den hiebei erzeugten Luft. druck numerisch zu bestimmen.

Der Kapillardruck differiert bei den verschiedenen Individuen auch bei normalen Hautverhältnissen. $\mathrm{Er}$ ist von verschiedenen Faktoren, die mit dem Kreislauf zusammenhängen, abhängig, die ich bei meinen Untersuchungen deshalb vernachlässigen kounte, weil ich bei jeder Messung einer veränderten Hautstelle den Kapillardruck der entsprechenden symmetrischen gesunden Hautstelle bestimmte und so bei jedem einzelnen Fall den Unterschied zwischen dem Kapillardruck der normalen und veränderten Haut numerisch feststellte.

Nur bei der Untersuchung der Urticaria-Quaddeln mußte ich davon abweichen, da nach Messung des Kapillardruckes der Quaddel, das aufgesetzte Trichterchen auch auf unveränderte

1) Prof. S. v. Basch. Ü̉ber die Messung des Kapillardruckes am Menschen und deren physiologische und klinische Bedeutung. Wiener klinische Rundschau. XIV, pag. 28. 
Haut gebracht, sofort durch den leichten Druck eine neue Quaddel hervorrief. Ich konnte daher erst nach Heilung der Urticaria den Kapillardruck der normalen Haut bestimmen, um ihn mit dem der Quaddel vergleichen zu können.

Ich untersuchte 1. acutes Ekzem, 2. chronisches Ekzem, 3. Herpes zoster, 4. Lues, 5. Urticaria.

Nach längerer Übung gelingt es leicht, genau zu bestimmen, in welchem Moment die betreffende Hautstelle gleichmäßig blaß wird, und dann am Manometer die diesem Druck entsprechende Zahl abzulesen.

1. A cutes $\mathrm{Ekzem}$. Ich beobachtete den Kapillardruck an $10 \mathrm{Fällen}$, bei deren jedem ich wiederholte Messungen vornahm. Stets war der Kapillardruck an den ekzematösen Stellen höher, als an den entsprechenden gesunden Hautstellen desselben Individuums.

$\begin{array}{ccccc}\text { Kapillar-Druck bei } & \text { Eez. acut. } & \text { gesunde Haut } & \text { Differenz } \\ \text { Fall } & \text { I } & 29 & 24 & 5 \\ \text { Fall } & \text { II } & 40 & 30 & 10 \\ \text { Fall } & \text { III } & 36 & 27 & 9 \\ \text { Fall } & \text { IV } & 32 & 26 & 6 \\ \text { Fall } & \text { V } & 29 & 23 & 6 \\ \text { Fall } & \text { VI } & 34 & 29 & 5 \\ \text { Fall } & \text { VII } & 36 & 28 & 6 \\ \text { Fall VIII } & 38 & 27 & 11 \\ \text { Fall } & \text { IX } & 28 & 24 & 4 \\ \text { Fall } & \text { X } & 33 & 25 & 8\end{array}$

Daraus folgt, daß sich als Durchschnitts;Differenz zwischen der akut ekzematösen und der gesunden Haut 7 Teilstriche ( $\mathrm{Mm}$. Hg) ergeben.

Im weiteren Verlaufe konnte ich während der Behandlung und Heilung des Ekzems die Abnahme des Kapillardruckes beobachten. Schon nach eintägiger Burow-Behandlung, die bei den akuten Ekzemen die häufigste war, sank der Kapillardruck an den ekzematösen Stellen um 
einige Teilstriche des Manometers. Bei Fall I war der Kapillardruck des noch unbehandelten Ekzems 29 (der Kapillardruck der gesunden Haut 24). Am nächsten Tage schwankte der Kapillardruck des durch Umschläge mit Liquor Burowi bedeutend gebesserten Ekzems zwischen 25-26 (gegen 24 der gesunden Haut). Im weiteren Verlauf war die Rückkehr des Kapillardruckes zur Norm eine ganz allmähliche.

Bei allen akuten Ekzemen konnte ich beobachten, daß die Abnahme des Kapillardruckes bei antiphlogistischer Behandlung in den ersten Tagen eine bedeutend größere war, als im weiteren Verlauf. Bei dem oben erwähnten Fall I war der Kapillardruck nach dem 1. Behandlungstag von 29 auf 26 gefallen, blieb dann 4 Tage ungefähr auf gleicher Höhe, schwankte 5 Tage zwischen $25-26$ und wurde dann innerhalb weiterer 3-4 Tage dem entsprechenden normalen Kapillardruck von 24 gleich.

Die Kurve, welche die Rückkehr des erhöhten Kapillardruckes zum normalen anzeigt, hatte folgenden Verlauf :

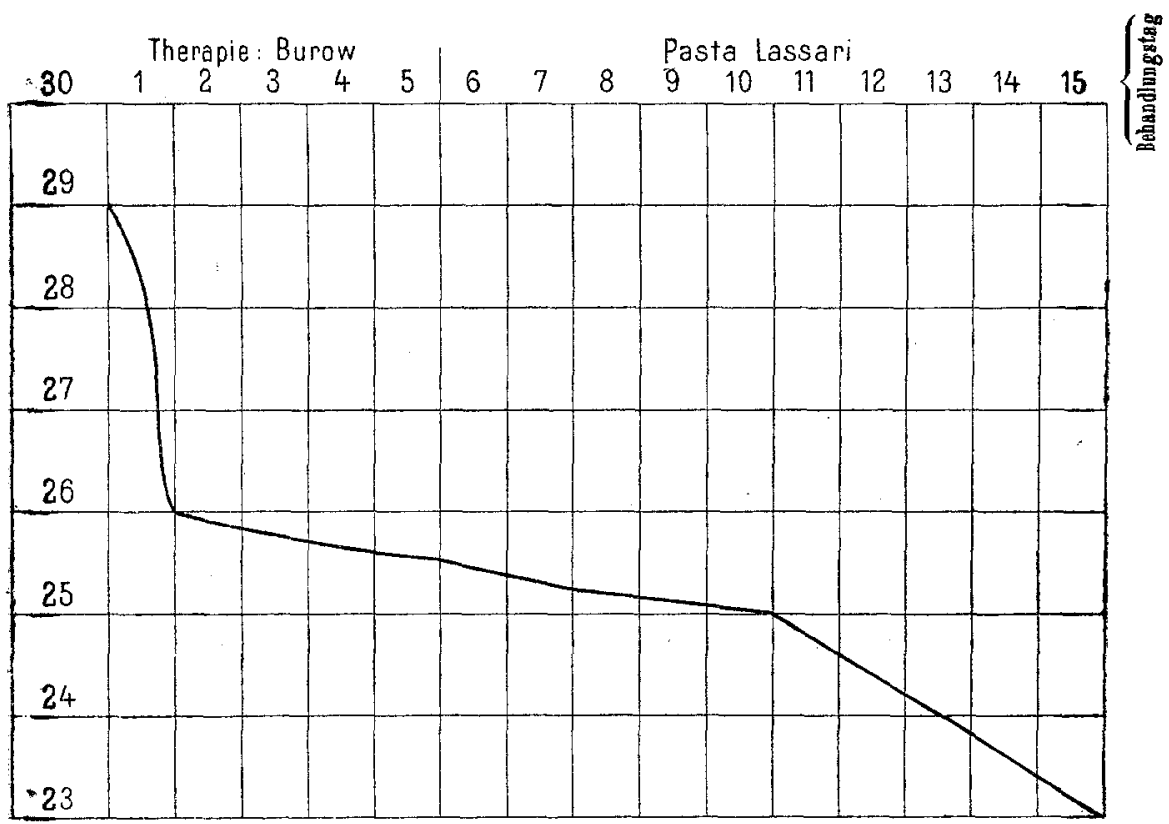

2. Beim chronischen Ekzem waren die Werte des Kapillardruckes an den ekzematösen Hautstellen geringer als an den entsprechenden gesunden Hautstellen. Es ist hier das chronische Lokalekzem gemeint, welches sich aus nicht ganz abgelaufenen Ekzemen oder nach wiederholtem Auftreten der akut ekzematösen Erscheinungen entwickelt. Das Blaßwerden der chronisch ekzematösen Haut war hiebei stets durch 
Über Kapillardruck-Messungen normaler v. veränd. Haut. 345

einen geringeren Druck zu erzielen, als das Blaßwerden der gesunden Hantstelle.

Chron. Eezem Gesunde Haut Differenz

$\begin{array}{lrll}\text { Fall } & \text { I . . . 20 } & 25 & 5 \\ \text { Fall II . . 18 } & 24 & 4 \\ \text { Fall III . . 22 } & 26 & 4 \\ \text { Fall IV . . 23 } & 25 & 2 \\ \text { Fall V . . . 21 } & 27 & 6 \\ \text { Fall VI . . . } 19 & 23 & 4 \\ \text { Fall VII . . . 23 } & 28 & 5\end{array}$

Durchschnittlich war der Kapillardruck der chronisch ekzematösen Hautstellen um 4 Theilstriche des Manometers geringer als der der gesunden Haut.

Ein 25jähr. Terpentinarbeiter, der mit einem akuten artefiziellen Ekzem beider Vorderarme in Behandlung stand, hatte bei einem Kapillardruck von 25 auf gesunder Haut, auf der akut ekzematösen Haut einen erhöhten Kapillardruck von 32. Nach dreiwöchentlicher Behandlung und bedeutender Besserung - der Kapillardruck war auf 26-27 gesunken blieb der Patient aus der Behandlung aus. 2 Wochen später kam Patient, welcher mittlerweile wiederholte ekzematöse Nachschübe gehabt hatte, wieder u. zw. mit dem klinischen Bilde des chronischen Ekzems. gesunken.

Der Kapillardruck der ekzematösen Hautstellen war bis auf 21

Auch an allen chronischen Unterschenkelekzemen, sowie in der Umgebung alter Unterschenkelgeschwüre war der Kapillardruck erniedrigt.

3. Herpes Zoster. Auf der geröteten Basis vor der Herpes zoster-Eruption, sowie an den geröteten Hautstellen in der Ungebung des bereits bestenden Herpes zoster fand ich erhöhten Kapillardruck. Ich beobachtete 5 Fälle:

Herp. zoster Gesunde Haut Differenz

$\begin{array}{lrrr}\text { Fall I. . . 30 } & 24 & 6 \\ \text { Fall II . . .27 } & 23 & 4 \\ \text { Fall III . . .38 } & 24 & 14 \\ \text { Fall IV . . . } 29 & 25 & 4 \\ \text { Fall V . . . 31 } & 24 & 7\end{array}$


Es hatten also die geröteten Stellen einen durchschnittlich um 7 Teilstriche des Kapillar-Manometers erhöhten Kapillardruck im Vergleich zu der gesunden Hant desselben Individuums.

Die Rückkehr zum normalen Kapillardruck war langsam und allmähỉch, synchron mit dem allmählichen Abheilen des Zosters. Waren zugleich mit der Eruption starke Entzündungserscheinungen vorhanden, wie bei Fall III (was aus dem hohen Kapillardruck - 38 gegen 24 der gesunden Haut - zu ersehen ist), so sank, wie beim akuten Ekzem, der Kapillardruck unter antiphlogistischer Behandlung in den ersten Tagen bedeutend, um dann ganz allmählich zur Norm zu gelangen.

4. Bei den Kapillardruck-Messungen der Lu es maculos a wählte ich zur Untersuchung reine makulöse Exantheme, deren Effloreszenzen unter Fingerdruck nahezu vollständig abblaßten. Ich fand stets erböhten Kapillardruck im Vergleich zur gesunden Haut. Die Erhöhung war hiebei stets bedeutender als die bei den vorgenannten Krankheitsformen beobachtete; d. h. der Druck, welcher notwendig ist, um beim makulösen Syphilid das gleichmäßige Blaßwerden der Macula zu erzielen, ist größer als der, welcher bei akutem Ekzem oder Herpes zoster zum Blaßwerden der Haut notwendig ist.

$\begin{array}{lccc} & \text { Macula luetica } & \text { Gesundo Haut } & \text { Differenz } \\ \text { Fall } & \text { I . . .37 } & 24 & 13 \\ \text { Fall } & \text { II . . .32 } & 21 & 11 \\ \text { Fall } & \text { III . . . 38 } & 26 & 12 \\ \text { Fall } & \text { IV . . . 35 } & 23 & 12 \\ \text { Fall } & \text { V . . . 40 } & 29 & 11 \\ \text { Fall } & \text { VI . . . 34 } & 22 & 12 \\ \text { Fall } & \text { VII . . . 37 } & 25 & 12 \\ \text { Fall VIII . . . } 41 & 27 & 13\end{array}$

Daraus folgt, daß der Kapillardruck der Effloreszenz durchschnittlich um 13 Teilstriche des Manometers höher war, als der der gesunden Haut.

Zugleich mit dem Abblassen des Exanthems nahm auch der Kapillardruck während der antiluetischen Behandlung allmählich $a b$, bis er beim Verschwinden des Exanthems dem der gesunden Haut gleich wurde.

5. Urticaria. Der Kapillardruck der Quaddel ist niedriger, als der Kapillardruck derselben Hautstelle, nach Ablauf der Urticaria gemessen. Der Druckunterschied ist allerdings nicht grob. 


$\begin{array}{lcc}\text { Fall I. . .20 } & 24 & 4 \\ \text { Fall II . . 21 } & 26 & 5 \\ \text { Fall III . . . } 19 & 23 & 4 \\ \text { Fall IV . . 22 } & 25 & 3 \\ \text { Fall V. . . } 23 & 27 & 4\end{array}$

Der Kapillardruck der Quaddel ist daher durchschnittlich um 4 niedriger als der der gesunden Haut.

Aus den vorstehenden Beobachtungen ergibt sich folgendes: Beim chronischen Ekzem und bei Urticaria ist der Kapillardruck der affizierten Hautstellen niedriger als der bei normaler Haut. Beimakuten Ekzem, sowie bei der mit akuten Entzündungserscheinungen einhergehenden Herpes zosterEruption, ferner bei luetischen makulösen Effloreszenzen ist der Kapillardruck erhöht.

Es ist also der Druck, welcher notwendig ist, um das Blut aus den hyperämischen feinsten Gefäßen zu vertreiben geringer bei chronischem Ekzem und Urticaria, größer bei akut entzïndlichen Erscheinungen der Haut und beim makulösen Syphilid.

Die Rückkehr des veränderten Kapillardruckes zur Norm erfolgt bei akut entzündlichen Erscheinungen anfangs sprungweise, indem am 1. oder 2. Behandlungstage unter antiphlogistischer Behandlung der Kapillardruck bedeutend sinkt, so daß er nur wenig rom Kapillardruck der normalen Haut differiert, dann aber ganz allmählich und langsam zur Norm zurückkehrt. Dies stimmt auch mit der klinischen Beobachtung überein, da ja bei den akuten Ekzemen der augenfälligste Teil der Besserung unter der antiphlogistischen Behandlung in den ersten Tagen wahrzunehmen ist, während die vollkommene Heilung allmählich eintritt und im Vergleich zum Ablauf der ersten Entzündungserscheinungen eine langsame ist. 
Bei allen anderen Messungen: dem chronischen Ekzem, Herpes zoster, der luetischen Macula, Urticaria wurde der Kapillardruck synchron mit dem allmählichen Verschwinden der Effloreszenzen, beziehungsweise der Rötung allmählich dem der entsprechenden normalen Haut gleich, und zwar rascher bei der Urticaria-Quaddel, entsprechend dem schnellen Verschwinden derselben, langsamer beim chronischen Ekzem und der luetischen makulösen Effloreszenz.

Vorstehende Untersuchungen machte ich auf Veranlassung des Herrn Abteilungsvorstandes Dozenten Dr. Spiegler, dem ich für die Anregung zu denselben herzlichst danke. 